\title{
Erratum to: Analysis of the Wsi18, a stress-inducible promoter that is active in the whole grain of transgenic rice
}

\author{
Nari Yi • Se-Jun Oh • Youn Shic Kim • \\ Hyun-Jun Jang $\cdot$ Su-Hyun Park · Jin Seo Jeong • \\ Sang Ik Song $\cdot$ Yang Do Choi $\cdot$ Ju-Kon Kim
}

Published online: 31 August 2010

(C) Springer Science+Business Media B.V. 2010

\section{Erratum to: Transgenic Res \\ DOI 10.1007/s11248-010-9400-y}

Due to an unfortunate mistake, the incorrect figure caption of Fig. 3 has been used in the above- mentioned publication. The correct representation of Fig. 3 and its caption are published on the following page and should be treated as definitive by the reader.

The online version of the original article can be found under doi:10.1007/s11248-010-9400-y.

N. Yi - S.-J. Oh · Y. S. Kim - H.-J. Jang - S.-H. Park · J. S. Jeong · S. I. Song · J.-K. Kim ( $\square)$

School of Biotechnology and Environmental Engineering, Myongji University, Yongin 449-728, Korea

e-mail: jukon306@gmail.com

N. Yi · Y. D. Choi

School of Agricultural Biotechnology, Seoul National

University, Seoul 151-921, Korea

S.-J. Oh

Syngenta Seeds Co., Ltd, 100 Gongpyeong-dong,

Seoul 110-702, Korea 

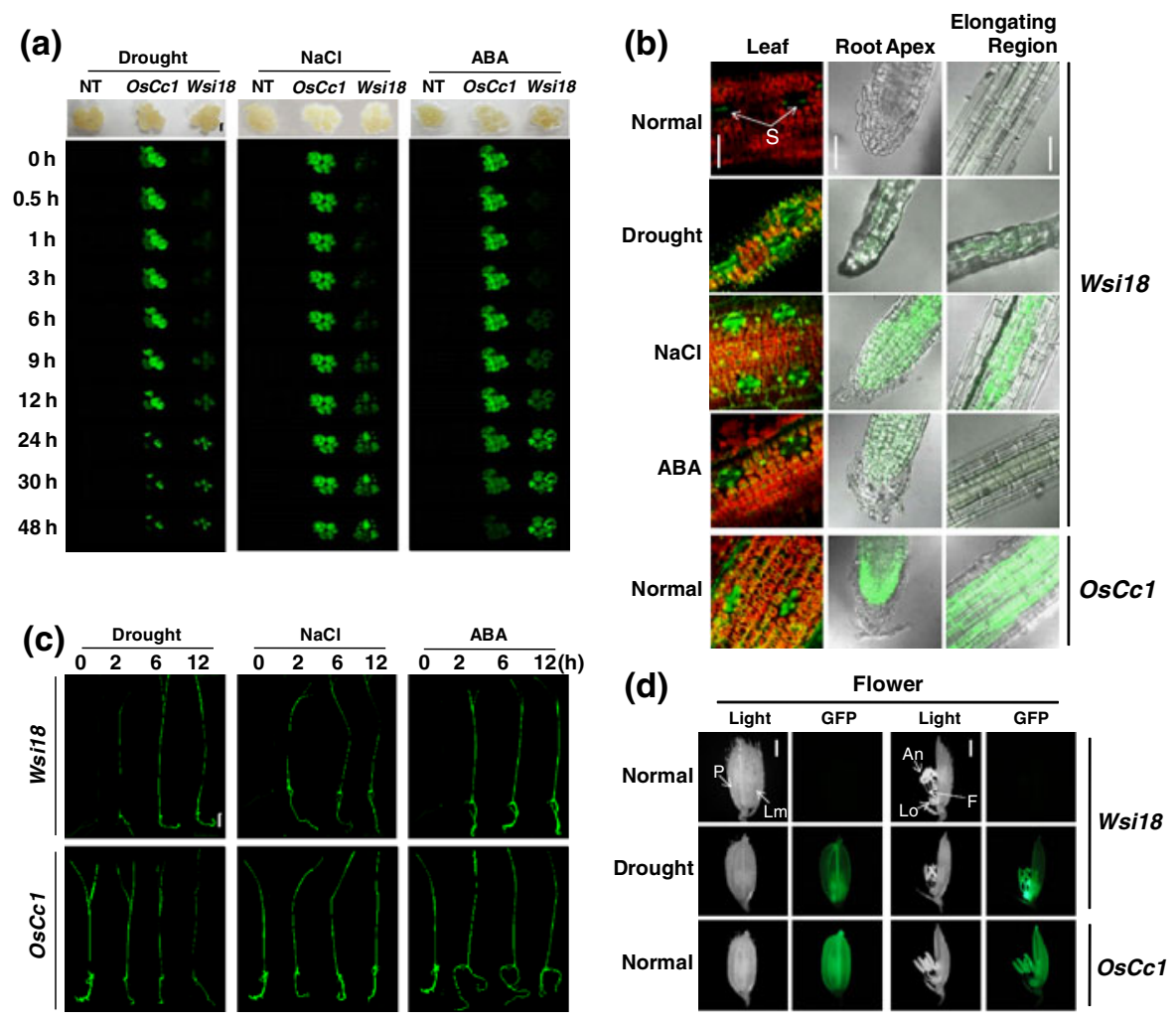

Fig. 3 Activity of the Wsi18 promoter in different tissues and growth stages of transgenic rice plants (line 2 shown in Fig. 2b). a GFP fluorescence in transgenic calli. GFP fluorescence was detected in calli exposed to drought, $\mathrm{NaCl}$ (400 mM), and ABA (100 lM) for the indicated time points using a luminescent-image analyzer. $N T$ non-transgenic calli, OsCcl calli induced from the OsCc1::GFP transgenic seeds (Jang et al. 2002), Wsil8 calli induced from the Wsi18::GFP transgenic seeds. Bar $=1 \mathrm{~mm}$. b Confocal microscopic images of GFP fluorescence in leaf and root tissues of transgenic plants. GFP fluorescence was detected in a leaf, a root apex, and a elongating region of a root of the Wsi18::GFP and OsCc1::GFP plants before (normal) and after exposure to drought, $\mathrm{NaCl}(400 \mathrm{mM})$, and $\mathrm{ABA}(100 \mathrm{lM})$ for $2-6 \mathrm{~h}$.

The images collected in the green and i channels of the confocal microscope were merged. Bars $=1 \mathrm{~mm}$. $S$ stomata. c GFP fluorescence in whole bodies of the Wsi18::GFP and OsCc1::GFP plants. GFP fluorescence was detected in 6-dayold etiolated plants exposed to drought, $\mathrm{NaCl}(400 \mathrm{mM})$, and ABA (100 lM) for the indicated time points using a luminescent-image analyzer. $B a r=1 \mathrm{~cm}$. d GFP fluorescence in transgenic flowers. GFP fluorescence was detected, by use of a stereomicroscope, in a whole spikelet (left panel) and floral organs inside a spikelet (right panel) at the stage of meiosis before (Normal) and after drought treatment. For drought treatment, water was withheld from plants in pots, in a greenhouse, for three days. An, anther; $F$, filament; $L m$, lemma; Lo, lodicule; $P$, palea. Bars $=1 \mathrm{~mm}$ 